\title{
Small angle X-ray scattering (SAXS) studies of sol to gel transition in $\mathrm{K}_{2} \mathrm{O}-\mathrm{Al}_{2} \mathrm{O}_{3}-\mathrm{SiO}_{2}$ system
}

\author{
K. J. LEE*, H. A. HRISTOV*, T. Y. TIEN*, E. GULARI** \\ *Department of Materials Science and Engineering and **Department of Chemical \\ Engineering, University of Michigan, Ann Arbor, MI 480109-2136, USA
}

The structural evolution during a sol to gel transition reaction in the $\mathrm{K}_{2} \mathrm{O}-\mathrm{Al}_{2} \mathrm{O}_{3}-\mathrm{SiO}_{2}$ system was investigated by using in situ small angle $\mathrm{X}$-ray scattering (SAXS) technique. The results are interpreted as evidence of the presence of at least two different gel phases. The analysis shows that the primary phase is composed of small particles with characteristic size of $\sim 3.0 \mathrm{~nm}$, and considerable size and shape variation. The primary phase is identified as a random phase in the Debye sense. The primary particles aggregate into larger formations with spherical symmetry. The size of larger aggregates (second phase) increases continuously in the course of gelation and levels off after reaching $\sim 15.0 \mathrm{~nm}$. The volume fraction of the second phase keeps increasing until the end of the measurement which is far after the gelation (3-fold of the gelation time).

\section{Introduction}

It is well known that a sol to gel reaction undergoes a series of chemical reactions namely, hydrolysis and polycondensation in the alkoxides system [1-3]. The gels resulting from these reactions may differ in structure and therefore in properties, depending on the experimental conditions [4-7]. The experimental variables such as the solvent, catalyst, water, reaction temperature, etc. should be optimized in order to produce gels with desired final properties. If one needs to control the final structure it is important to investigate the effect of the variables on the formation of gels. In order to properly assess these effects, it is crucial to obtain detailed information on the structural evolution during a sol to gel transition reaction.

Various scattering techniques have been used [8-11] to characterize the evolution of the sol-gel reaction in terms of the size of the growing gel particles. It has been established that light scattering $[8,9]$ including Raman scattering [10], and small angle X-ray scattering (SAXS) [11] are the most useful techniques in this respect. Transmission electron microscopy (TEM) measurements of gels, frozen at various stages of the sol-gel transformation, have been reported on a few occasions $[12,13]$. Light scattering is employed mainly to investigate macromolecules in solution. The size resolution of the light scattering methods is several hundreds of angstroms, thus the method cannot be used to characterize the early stages of the sol-gel transformation (where the particle size is less than $10.0 \mathrm{~nm}$ at this stage [11]). Raman scattering techniques have been successfully employed to follow the kinetics of the reaction at later stages.

The X-ray scattering at small angles results from fluctuations of the electronic density within the material with dimensions in the range $2.0-200.0 \mathrm{~nm}$ [14].
Recently, the SAXS technique has gained in popularity in conjunction with fractal concepts $[11,15,16]$. It was established that some systems exhibit a scaled self similarity of the main building units. In order to obtain the necessary information, the gelation process is usually interrupted at specific time intervals for dilution of the material, and postmortem investigation. While such studies provide valuable information with regard to the fractal character of the main building units, it is not evident that the results depict the real gel structure (a discussion of some problems can be found in [17]). Another problem associated with the dilution technique is the lack of information with regard to the kinetics of the process. A brief analysis of the sol-gel transition in undiluted systems is presented in [18] but this analysis appears to be incomplete since it does not account for the interference of the scattered intensity from neighbouring particles when the concentration/time increases; also the possible development of more than one gel phases is not taken into account. The same is valid for the analysis of the results presented in [11], however this work indicates the possibility of agglomeration of smaller building units.

In the present work we report an in situ small angle $\mathrm{X}$-ray scattering investigation of the sol to gel transition in the $\mathrm{K}_{2} \mathrm{O}-\mathrm{Al}_{2} \mathrm{O}_{3}-\mathrm{SiO}_{2}$ system. The composition was chosen to form a monolithic form of feldspar glass as reported in a previous paper [19]. The main goal of the investigation is to obtain quantitative information regarding the structural developments during the sol to gel reaction.

\section{Experimental procedure}

The starting materials used to prepare the alkoxide solution were potassium methoxide $\left(\mathrm{K}\left(\mathrm{OCH}_{3}\right)\right)$, 
aluminum sec-butoxide $\left(\mathrm{Al}\left(\mathrm{OC}_{4} \mathrm{H}_{9}\right)_{3}\right)$, and tetramethoxysilane (TMOS, $\left.\mathrm{Si}\left(\mathrm{OCH}_{3}\right)_{4}\right)$. Their molar ratios were adjusted to have a potash feldspar glass composition of $\mathrm{K}_{2} \mathrm{O}: \mathrm{Al}_{2} \mathrm{O}_{3}: 6 \mathrm{SiO}_{2}$. TMOS was dissolved in methanol and formamide was used as a drying control additive to prevent crack development during drying [20]. The mixture was vigourously stirred and $\mathrm{K}\left(\mathrm{OCH}_{3}\right)$ was then added to the solution. During the continuous stirring an appropriate amount of $\mathrm{Al}\left(\mathrm{OC}_{4} \mathrm{H}_{9}\right)_{3}$ was added. The mixing of the alkoxides was processed under a nitrogen atmosphere at $80^{\circ} \mathrm{C}$, which produces a clear solution. After cooling to room temperature, the solution was hydrolysed by the addition of distilled water. The sample solution was immediately injected in a specially designed stainless steel cell with a mylar ${ }^{\circledR}$ window (window thickness less than $0.1 \mathrm{~mm}$, sample thickness $\sim 2 \mathrm{~mm}$ ) which can be mounted on the SAXS instrument.

SAXS measurements were performed by using a diffractometer with a Kratky type collimation (Anton Paar, Austria). To obtain a sufficient size resolution $\mathrm{Fe}$ $K_{\alpha}$ radiation was used, collimated with an entrance slit of $30 \mu \mathrm{m}$. The diffracted radiation was registered by a position-sensitive proportional counter (M-Broun, Germany, Model OED 50) in $2 \theta$ interval from $0.06^{\circ}$ up to $6.6^{\circ}$ simultaneously in time intervals of $5 \mathrm{~min}$ utes. The angular interval corresponds to a reciprocal space interval from $k_{\min }=0.03 \mathrm{~nm}^{-1}$ to $k_{\max }=$ $3.7 \mathrm{~nm}^{-1}$, where $|\mathbf{k}|=4 \pi \sin \theta / \lambda$ is the usual diffraction vector, $\theta$ is the diffraction angle and $\lambda$ is the wavelength of the $\mathrm{Fe} K_{\alpha}$ radiation, $(0.194 \mathrm{~nm})$. The maximum size resolution of the instrument is in the range $200-300 \mathrm{~nm}$, depending on the scattering power of the material. The scattering measurements were performed at room temperature in situ throughout the sol to gel reaction. The intensities collected at different times were corrected for background and air scattering, and then desmeared (infinitely long slit approximation) [21].

\section{Results}

Fig. 1 shows a typical scattering curve of $\log$ (intensity) vs. $\log (k)$ obtained at the beginning of the sol to gel reaction when the system is still diluted $\left(t / t_{\mathrm{g}}=0.2\right)$. The result demonstrated that the tail is not linear, i.e. there is no simple power law applicable to our system. It has been found that in a dilute system of scatterers with identical (or narrow distribution) sizes and shapes, the decrease of the scattered intensity with increasing $k$ (or angle) follows a power law i. e. $I(k)$ $\times k^{n}=$ const. In the case of particles with "proper" shape (e.g. spheres, discs, rods) $n$ is an integer [21], while in the case of objects with irregular shape the $n$ value is often a fractal number [16]. Scattering results which exhibit significant curvature in a log-log plot have been reported for some silicate polymers [5], but the possible source of the nonlinearity has not been discussed. Deviation from the power law usually indicates scattering from dissimilar particles [14], thus the curve in Fig. 1 can be consistently explained if we assume that the scattering entities have significant variations of sizes and/or shapes. In such cases it may

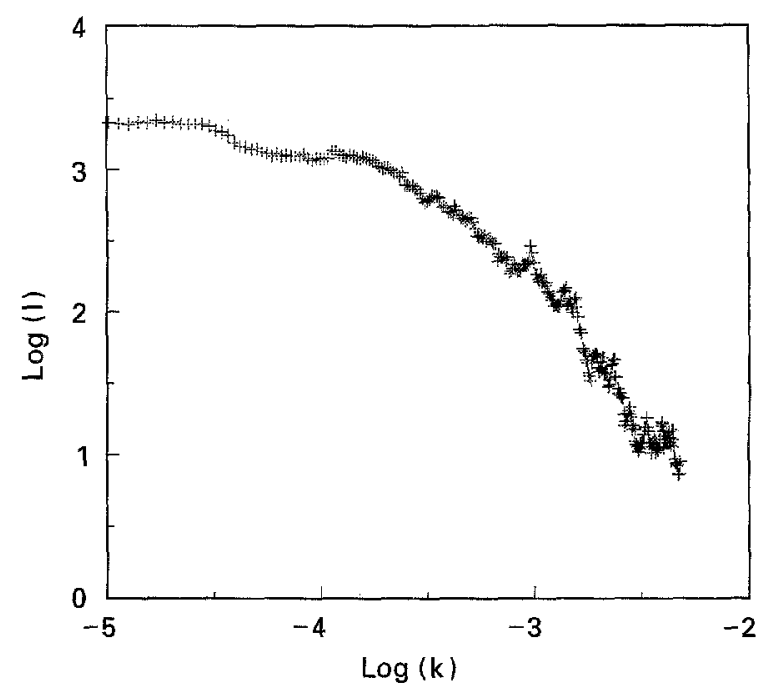

Figure $1 \log$ intensity vs. $\log k$ at reduced time, $t / t_{\mathrm{g}}=0.2$.

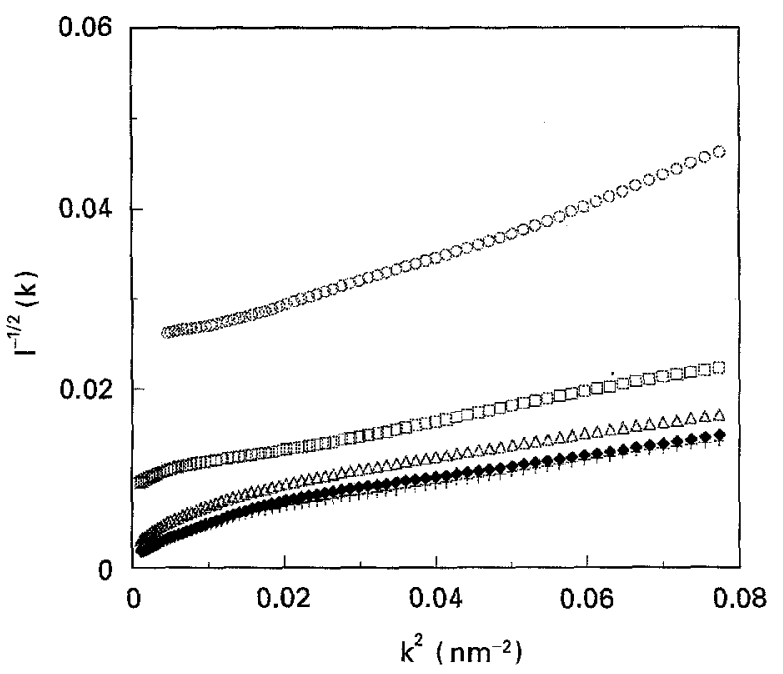

Figure 2 Debye plot of the intensities at different reduced reaction times; (O) $t / t_{\mathrm{g}}=0.2,(\square) t / t_{\mathrm{g}}=0.4,(\Delta) t / t_{\mathrm{g}}=0.8,(\bullet) t / t_{\mathrm{g}}=2.0$ and $(+) t / t_{\mathrm{g}}=3.0$.

be fruitful to attempt to analyse the diffraction curves as a scattering from a completely random system (zero order Poisson distribution [22] of particle sizes). According to Debye et al. [23] the correlation function, $\gamma(r)$, of such system is given by:

$$
\gamma(r)=\exp (-r / a)
$$

where $r$ is an arbitrary distance within the scattering medium and $a$ is the Debye correlation length.

Using the above correlation function, Equation 1, the $\mathrm{X}$-ray intensity diffracted from a random system can be expressed as [23]:

$$
I(k)=\frac{\text { constant }}{\left(1+k^{2} a^{2}\right)^{2}}
$$

If the electron density fluctuations in our system conform to Equation 1, then a plot of $I(k)^{-1 / 2}$ vs. $k^{2}$ should yield a straight line from Equation 2. As shown in Fig. 2, the intensities obtained at various times can be linearized when plotted as $I(k)^{-1 / 2}$ vs. $k^{2}$, and the obvious conclusion is that the system is random in the 


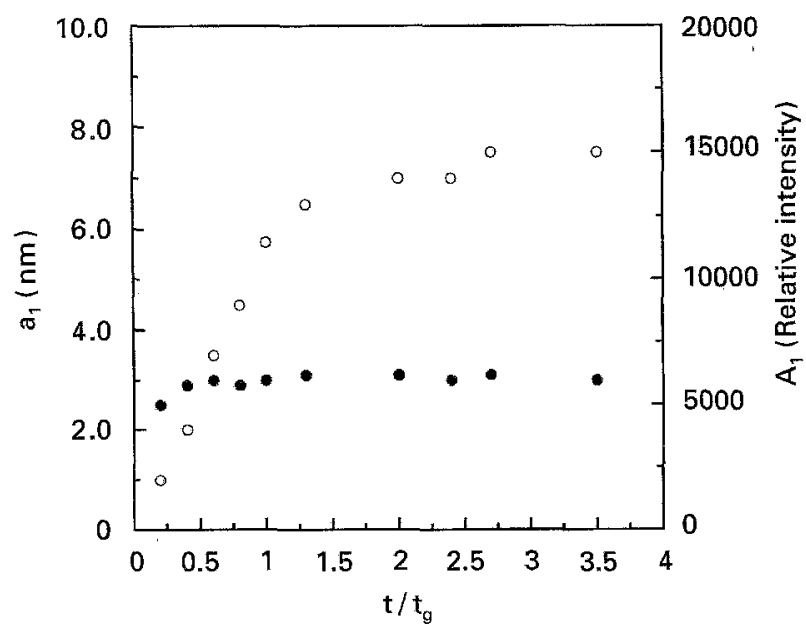

Figure 3 The change of the characteristic size $\left((\boldsymbol{O}) \mathrm{a}_{1}\right)$ and volume fraction ( $\left.(O) A_{1}\right)$ of the primary phase with reduced time.

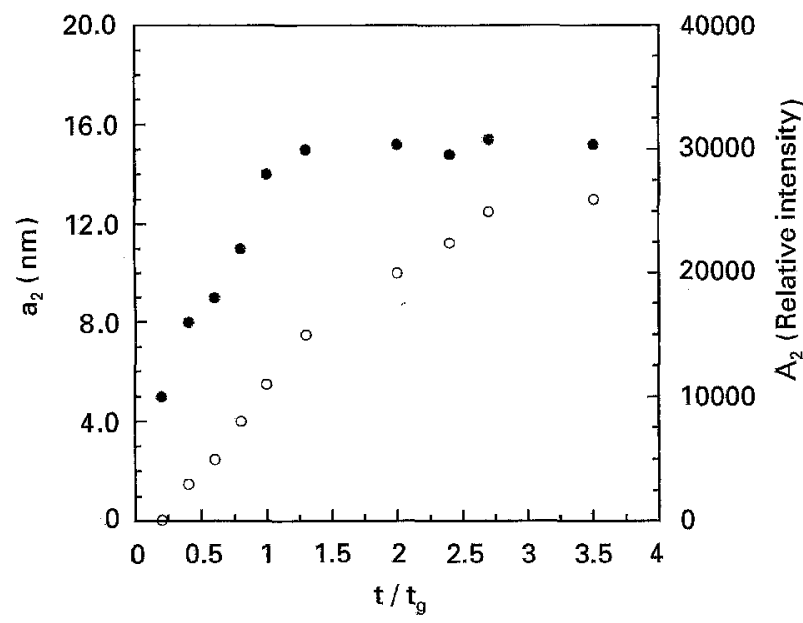

Figure 4 The change of the size $\left((\odot) \mathrm{a}_{2}\right)$ and volume fraction ((O) $\mathrm{A}_{2}$ ) of the second phase aggregations (see text for details).

Debye sense. An advantage of using the Debye approach is that the analysis is independent of the concentration of the scattering entities, as long as they remain random in size and shape. From Fig. 2, it is also possible to obtain quantitative information by deriving the characteristic length via the following relationship:

$$
a=\left(\frac{\text { slope }}{\text { intercept }}\right)^{1 / 2}
$$

Fig. 2 also shows that the scattering curves obtained at larger times exhibit a small drop-off from the straight line at low angles. In such cases, the scattering curves can be adequately corrected by including a second term in the correlation function [23] and the total intensity is written as:

$$
I(k)=A_{1}\left(1+k^{2} a_{1}^{2}\right)^{-2}+A_{2} \exp \left(-k^{2} a_{2}^{2}\right)
$$

where $a_{1}$ is the Debye characteristic length, $a_{2}=\left(R_{\mathrm{g}}^{2} / 3\right)^{1 / 2}$, respectively, with $R_{\mathrm{g}}$ the gyration radius of larger scatterers. The corrected experimental intensities were fitted with four parameters in accordance with Equation 4 and the results from this procedure are shown in Figs 3 and 4. The data points in each figure are accurate within $3 \%$.

\section{Discussion}

The interpretation of the second term in Equation 4 is the presence of a second phase in the gel, which appears to be more homogeneous in size and shape for a fixed time period. The amplitudes $A_{1}$ and $A_{2}$ (intensities at $k=0$ ) are related to the total amount of the two respective phases. Fig. 3 shows that the characteristic size $(\sim 3.0 \mathrm{~nm})$ of the random phase $\left(a_{1}\right)$ is essentially constant in the course of gelation, while its amount $\left(A_{1}\right)$ increases in a linear manner and reaches a plateau after the gelation point. It should be noted that the term "characteristic size" means that $\sim 3.0 \mathrm{~nm}$ is the most probable dimension, but one should expect a significant size variation within the random phase. The relatively stable characteristic size implies that the increase in $A_{1}$ is proportional to the increase of the volume fraction of the random phase, i.e. the volume fraction of the random phase increases linearly with time. Linear growth with time is usually interpreted [24] as an indication of instantaneous formation of random phase $\left(a_{1}\right)$. A consistent interpretation of this result is the assumption that the random phase is composed of small (primary) particles, which grow very rapidly (with respect to the measuring time of $1 \mathrm{~min}$ ) to their final size. The size of the larger scatterers (second phase, $a_{2}$ ) increases continuously in the same time interval and after reaching $\sim 15.0 \mathrm{~nm}$ levels off as shown in Fig. 4. The volume fraction of the second phase $\left(A_{2}\right)$ increases until the end of the measurements which is 3-fold of the gelation time. Since the increase of the average radius of gyration $\left(a_{2}\right)$ of the secondary particles is reasonably linear for $t<t_{\text {gel }}$, their growth before the gelation is apparently a three dimensional process.

The results discussed above suggest that the gelation process starts with the formation of small primary particles, which grow very rapidly. It is reasonable to expect that rapid growth will produce formations with considerable size and shape variations. As the number of primary particles increases steadily, they tend to aggregate forming larger scatterers in random localities throughout the sample volume. The aggregates of these primary particles grow in size and number as the sol to gel reaction progresses, until they reach a final size of $\sim 15.0 \mathrm{~nm}$ after the gelation point. At the gelation point, the gel phase has lost the ability to flow freely but the system still has the sol fraction which is available for further growth. It was found that the alkoxide was not totally consumed until about the gelation point when Raman spectroscopy was used to follow the disappearance of TMOS as reported in a previous study [19]. The hydrolysis of the alkoxides and condensation of the hydrolysed species produce the primary particles. However, the volume fraction of the primary particles stops increasing soon after all the alkoxides are consumed. This eventually limits the growth of the aggregate size to $\sim 15.0 \mathrm{~nm}$. It is clear that both hydrolysis and growth proceed at the same time, and the condensation develops mainly by the growth of hydrolysed species. It is evident also (Fig. 4) that the agglomeration process continues well after the gelation time, although the rate of formation of secondary particles decreases. 
The two phase structural development proposed in our work is consistent with results from TEM investigations of frozen silica gels $[12,13]$, and postmortem SAXS study of fired silica gels [25]. Because of the nature of these investigations the kinetics of the gelation process is not known, but the structural results indicate the formation of small particles with sizes in the range $2.0-4.0 \mathrm{~nm}$, which probably agglomerate in a later stage. The size of the secondary particles reported in $[25](\sim 100.0 \mathrm{~nm})$, appears to be an order of magnitude larger than the sizes measured in our study. It should be noted that the random structure model proposed by Debye et al. [23], has been used before to analyse the structure of silica gels [25], but the present study is its first application as a tool to follow the kinetics of the sol to gel transformation on a microscopic scale.

Finally it is useful to discuss the random structure from a fractal point of view. If we rewrite Equation 2 as $I(k)=$ const $/\left(1+2 k^{2} a^{2}+k^{4} a^{4}\right)$ it is evident that at small $k$ (low angles) the $k^{-2}$ term will dominate the intensity distribution. At large $k$, the $k^{-4}$ term (Porod term) will determine the shape of the scattering curve, while at intermediate angles, different small portions of the curve can be fitted with $(k a)^{-n}$, where $n$ is any value between two and four (including fractal values). The simple considerations outlined above indicate that only measurements in a sufficiently large angular interval can determine whether a particular system is random or exhibits some scaling property. When attempting to fit the tails of the scattering curves (i.e. at high angles) by a power law, one should also correct the "parasitic" contribution from the wide angle scattering since it may affect the value of the exponent. In our opinion these questions have not been adequately addressed in the specialized literature.

\section{Conclusions}

It is demonstrated that the structural evolution during a sol to gel transition reaction in $\mathrm{K}_{2} \mathrm{O}-\mathrm{Al}_{2} \mathrm{O}_{3}-\mathrm{SiO}_{2}$ system can be characterized by using in situ small angle X-ray scattering techniques. The results show that the final gel structure is composed of at least two different phases. The primary phase is composed of small particles (characteristic dimension $\sim 3.0 \mathrm{~nm}$ ) exhibiting a considerable size and shape variation. This phase possess the characteristics of a completely random material. The characteristic dimension of these particles is essentially constant before the gelation, while its volume fraction increases linearly in the same time interval. The nucleation of primary particles ceases soon after the gelation point. The primary particles aggregate during the course of reaction. The size of the aggregates (second phase) increases linearly until the gelation time, reaches gyration radius of $\sim 15.0 \mathrm{~nm}$ and levels off. The volume of the second phase increases until the end of the measurement which is far after the gelation (3-fold of the gelation time).

\section{Acknowledgement}

This research was partially supported by NIH through DE-09296.

\section{References}

1. R. ROY, J. Amer. Ceram. Soc. 52 (1969) 344.

2. B. E. YOLDAS, J. Mat. Sci. 14 (1979) 1843.

3. R. C. MEHROTRA, "Polymetallic Alkoxides - Precursors for Ceramics," Mat. Res. Soc. Symp. Proc. 121 (1988) 81.

4. B. E. YOLDAS, J. Non-Cryst. Solids 83 (1986) 375.

5. K. D. KEEFER, Mat. Res. Soc. Symp. Proc. 32 (1984) 15.

6. H. KOZUKA, S. H. KIM and S. SAKKA, in Ultrastructure Processing of Advanced Ceramics, Edited by J. D. Mackenzie and D. R. Ulrich, Wiley, New York (1988) pp. 159-81.

7. C. J. BRINKER, J. Non-Crystalline Solids 100 (1988) 31.

8. C. H. BYERS and M. T. HARRIS, in Ultrastructure Processing of Advanced Ceramics, Edited by J. D. Mackenzie and D. R. Ulrich, Wiley, New York (1988) pp. 843-53.

9. E. C. ZIEMATH, W. L. PRETTI, M. A. AEGERTER, J. E. C. MOREIRA, T. LOURS and J. ZARZYCKI, J. NonCryst. Solids 100 (1988) 93.

10. T. W. ZERDA, M. BRADLEY and J. JONAS, Mat. Lett. 3 (1985) 124.

11. T. LOURS, J. ZARZYCKI, A. CRAIEVICH, D. I. DOS SANTOS and M. AEGERTER, J. Non-Cryst. Solids: 100 (1988) 207.

12. J. K. BAILEY and M. L. MECARTNEY, Mat. Res. Soc. Proc. 121 (1988) 367.

13. Idem, ibid. 180 (1990) 153.

14. A. GUINIER and G. FOUR NET, in Small Angle Scattering of X-Rays, Wiley (1955).

15. D. W. SCHAEFER and K. D. KEEFER, Mat. Res. Soc. Symp. Proc. 73 (1986) 277.

16. Idem, ibid. 73 (1986) 295.

17. C. J. BRINKER, K. D. KEEFER, D. W. SCHAEFER, R. A. ASSINK, B. D. KAY and C. S. ASHLEY, J. Non-Cryst. Sol. 63 (1984) 45.

18. D. W. SCHAEFER and K. D. KEEFER, Mat. Res. Soc. Symp. Proc. 32 (1984) 1.

19. K. J. LEE, T. Y. TIEN and E. GULAR I, J. Non-Cryst. Solids 171 (1994) 46.

20. G. ORCEL, J. L. NOGUES and L. L. HENCH, Mat. Res. Soc. Symp. Proc. 73 (1986) 35.

21. O. GLATTER, O. KRATKY, in Small Angle X-Ray Scattering, Academic Press (1982) pp. 18-51.

22. F. J. BALTA-CALLIJA and C. G. VONK, in X-ray Scattering of Synthetic Polymers, Elsevier (1989) pp. 259.

23. P. DEBYE, H. R. ANDERSON, Jr and H. BRUMBERGER, J. Appl. Phys. 28 (1957) 679.

24. M. AVRAMI, J. Chem. Phys. 7 (1939) 1103; 8 (1940) 212; 9 (1941) 177.

25. I. M. DRAKE, P. LEVITZ and S. SINHA, Mat. Res, Soc. Symp. Proc. 73 (1986) 305.

Received 21 September 1994

and accepted 8 September 1995 